\title{
Erratum to: Childhood and Tween Girl Culture
}

\section{Fiona MacDonald}

Erratum to:

Childhood and Tween Girl Culture, Studies in Childhood and Youth DOI 10.1057/978-1-137-55130-6

The 'Acknowledgement' section in the front matter, which was missing in the original version has been included.

The online version of the original book can be found under DOI 10.1057/978-1-137-55130-6

Fiona MacDonald

Monash University

Victoria, Australia 
